\title{
Introduction to Statistical Physics outside Physics
}

\author{
Dietrich Stauffer \\ Institute for Theoretical Physics, Cologne University \\ D-50923 Köln, Euroland
}

\begin{abstract}
We review the possibilities and difficulties for statistical physicists if they apply their methods to biology, economics, or sociology. WARNING: I report opinions, not simulations.

Keywords: Biophysics, econophysics, sociophysics.
\end{abstract}

\section{Introduction}

The basic theorem of interdisciplinary research states: Physicists not only know everything; they know everything better. This theorem is wrong; it is valid only for computational statistical physicists like me. (Some conference speakers still seemed to believe in the theorem; they can discuss it in their papers.) Here I present my personal view about the application of statistical physics (simulation) methods to fields outside physics like biology, economic, sociology and even psychology.

\section{$2 \quad$ Possible criticism}

Typical criticisms levelled against physicists outside physics are "Biology is more complicated than physics, physicists neglect details." True; but the Earth is more complicated than a point mass; nevertheless Kepler's laws are quite accurate and were very useful. Physicists should try to find the simplest model giving the desired result, and not to make the model more realistic only for the sake of realism. Of course, Kepler knew that the Earth was not a point. He knew of the 30-year war destroying central Europe during his later life, for he was an unlucky astronomer advising general Wallenstein who was murdered. Kepler died when he tried to get his astrologer's salary from the imperial parliament. So, Kepler could have tried to build the large size of the Earth into his description, to deal with the different countries of Europe, and to understand the religious differences which caused the war to start. But this would have been a hindrance, not a help, for the understanding of planetary motion. Only much later, thanks to Newton and others, did mankind understand that a spherically extended mass has a gravitational field equal to that caused if the whole mass would have compressed to a point in the center of the sphere. And even later, thanks to Einstein's General Relativity Theory, did experts understand how the mass affects the metric of time and length through curved space. For the planetary motion, Einstein's theory then explained minor deviations from Kepler's laws 
like the rotation of Mercury's perihel. Perhaps the now started century will explain how the Higgs particle produces the mass, and bring new effects. But the basic Kepler laws from around 1610 are still the starting point of modern physics. Of course, if you want to be a geographer, the assumption of a point-like Earth is dangerous for your employment: The same model may be good for some and bad for other purposes.

"Computers know only 0 and 1." Wrong; many shades of grey are possible, and integers on 32-bit computers vary between -2147483648 and +2147483647 . I don't know a human being who can distinguish anything with an accuracy of $10^{-9}$. But when dealing with a new problem, why not first assume that the state variables can only be +1 and -1 ( 1 and 0 for informatics). Monte Carlo simulations with discrete Ising models can be done for thousand times bigger systems than in molecular dynamics studies on a continuum $\left(10^{13}\right.$ versus $\left.10^{10}\right)$. Whoever studied newspapers in the last decades came across the difficulties of defining when a person is dead, allowing the organs to be transplanted. Our hair and nails still grow long after our hearts stop beating; some readers of my papers claim my beer belly grew after my brain stopped working. Nevertheless, newspapers report the death of a person, and statistical offices prepare life tables as if life $(+1)$ and death $(-1)$ are the only possibilities without anything in between. It is animals like Caenorhabditis elegans with its "dauer" state (some sort of hibernation) for which intermediate states are very important, not human beings. Finally, many social problems are connected with more than one variable, but so are many models of physicists. Seven-dimensional percolation and five-dimensional Ising models show that physics is not one-dimensional, and neither is immunology where a a shape space of high dimensions was suggested already in 1979 before such models could easily be simulated. (In contrast to widespread belief, the Ising model does not only allow +1 and -1 as state variables. Much work has been done on Blume-Capel-Rys-Blume-Emery-Griffiths models where also zero is allowed.)

"Humans are not numbers." Wrong; we just don't want to be treated as numbers, and prefer a name tag instead of a number for identification. But reality is different: US citizens have a social security number, I have a national identity card with a computer-readable number; only with a computer-readable passport can I now enter the USA; my employer, my health insurance and my bank all gave me a number. Whether I smoke, drink wódka in the morning, and eat steaks every evening influences my date of death, and neither employer nor health insurance know about it. Nevertheless, by averaging over millions of people, these personal details cancel out. Similarly, the decision to get a child is highly private, but nevertheless I am most likely right if I claim that in 2004 hundreds of thousands of children will be born in Germany. If today's pension plans are in trouble it is because the average life expectancy increases and the average number of births per women decreased, not because of individual fluctuations. Thus humans and atoms may be described by the same method, if we look at averages. And besides the above rather recent examples we have astronomer Halley trying to establish the first human life tables three centuries ago, and Greek philosopher Empedokles 
claiming more than two millennia ago (according to Mimkes) that some people are like water and wine, mixing easily, while others are like water and oil, not mixing.

"Physics is different from Sociology and/or Economy." True; but Schelling [1] in first issue of J. Mathematical Sociology simulated something like the dilute Ising model with Kawasaki kinetics to explain black ghettos in USA; and Nobel laureate Stigler [2] published market simulations in 1964 before any conference participant wrote the first simulation paper. So why cannot we follow them? Indeed, our problem is much more than we may not know such pioneering papers in non-physics journals, and thus erroneously believe that we bring new concepts to such fields with our physics experience.

\section{Do other fields welcome physicists?}

From my own experience in bio-, econo- and socio-physics my answer depends on the field; psychophysics [3] is too new for me.

\subsection{Economics: yes}

Economy Nobel laureate Harry Markowitz [4] wrote: "I believe that microscopic market simulations have an important role to play in economics and finance. If it takes people from outside economics and finance - perhaps physicists - to demonstrate this role, it won't be for the first time that outsiders have made substantial contributions to these fields." Economics professor Thomas Lux knows a lot about physics; one we discussed whether to do a Grassberger-Procaccia analysis of some financial data and then I realized that in this case he, the economist, would have to explain to me, the physicist, how to apply this physics method. Economics professor Haim Levy wrote papers and a book with theoretical physicist Sorin Solomon and with his son Moshe Levy, a doctoral student of physics who is now economics professor.

Kertész reported that at a recent econophysics conference the economists criticized the physicists for re-inventing the economic wheels. This I count as acceptance, not as rejection: If A claims do have done first what B reports now, then A regards this B problem as important. Somewhat similarly, when in our university magazine I reported about Ehrenstein's simulations of the Tobin tax, a senior economics professor criticized me, perhaps correctly, for having ignored the dangers of government currency controls, as Germany had it during the Nazi dictatorship; again, the problem was taken serious by an economist. 


\subsection{Sociology: yes, if computational sociologists}

A year after I started to simulate Sznajd models, I presented a review at the fifth conference on simulating society, SIMSOC V, in Poland, organized by psychologist A. Novak. I felt quite at home with some of the talks of non-physicists, with simple models giving clear results. In contrast the international Physics Computing 2001 meeting two weeks earlier was full of complicated simulations understandable to specialists only. I learned from Hegselmann's talk (from a philosophy department) about bounded confidence and used that in several later papers of mine. Of course, for a conference on "Simulating Society" the participating sociologists are a highly biased selection.

An important problem is how to get data, which then should be modeled by sociophysics. Election results, more precisely the distribution of votes among many candidates, have been modeled successfully for both Brazil and India. This example [5] also shows the limitations: The methods of statistical physics can predict the probability distribution functions for the velocity of air molecules and the election votes, but they cannot predict where a specific air molecule will be a second later or which candidate will win the election. The internet allows physicists and computer scientists to collect lots of data on who is sending e-mail to whom, which web site cites another web site, and which computers are directly connected to other computers. These evolving networks yield much better statistics than the less than 100 people at the South Pole recently investigated for social roles and leadership [6], ignoring the recent network literature in physics.

\subsection{Biology: no, but changing}

Traditionally, the only good biology was experimental biology, a view also held by famous interdisciplinary physicist H.E. Stanley (whom I had to replace by this talk) who analyzed empirical data from biology and economics. But with billions of base pair data available now from DNA analysis of the whole genome, the need to interpret these biological data arises. Spin glass physics taught us that knowing all the interactions between the spins and all the spin orientations does not yet solve the problem how the spin glass behaves as a whole. Similarly, bio-informatics needs to understand how to deduct from the known DNA the behaviour of the organism. Today, nobody has as yet produced a living being out of non-living matter. Thus biology should take modelling more serious than in past decades, and I see some changes in the behaviour of young biologist. After all, I became a member of the advisory board of one biological and one medical journal, never having followed a university course in these fields.

What we need in biologically motivated simulations are real successes. There are lots of papers on theoretical immunology, but vaccination works since more than a century without needing my excellent simulations. Ageing still cannot be prevented, in spite of experimental 
and theoretical work on it. A big effect, comparable to the atomic bomb of 1945, is still lacking in theoretical biology.

\subsection{Econo-bio-socio-physics ?}

Of course, even more difficult is to become accepted by work touching on all three of the above fields, like predicting the "age quake" around 2030 when the 70 -year old people may be the strongest age cohort in rich countries. Should they trust existing pension regulations or build up their own savings for old age? Politicians debate this hotly in several countries of the European Union. Thus far none of them asked for my advice.

\section{Acceptance time}

Let us assume that these interdisciplinary attempts of physicists are objectively good. How long do we have to wait until they will be accepted by the other field? After one decade of ageing simulations by physicists, a few biologists have started to cite them. A dozen years after the car traffic models of Nagel and Schreckenberg as well as Biham, Middleton and Levine, I don't see yet applications in many cities and on many expressways, but serious application effort exists in Duisburg (Germany), Dallas (Texas) and Portland (Oregon). In 1976 the later Nobel laureate de Gennes was one of those who suggested that the critical exponents of threedimensional percolation should be found in real gelation experiments. This suggestion was far from revolutionary since the later chemistry Nobel laureate Flory invented percolation theory in 1941 to explain gelation (Bethe lattice, same universality class as random graphs or other meanfield approaches). Nevertheless it took more than a decade before chemistry experimentalists widely accepted this idea. Thus, for fields much further apart, like physics and sociology, we should measure the times for possible acceptance in decades, not in months. Similarly, people planting trees for a forest work for future generations.

\section{Summary}

Exotic physics has become less exotic at this conference but it still rejected by many, both within and outside physics departments. But there is some consolation: The gap between theoretical physicists and theoretical biologists (sociologists) may be smaller than that between the latter ones and experimental biologists (sociologists). 
[1] T.C. Schelling, J. Mathematical Sociology 1 (1971) 143.

[2] G.J. Stigler, Journal of Business 37 (1964) 117.

[3] L. da Fontoura Costa, cond-mat/0309266.

[4] S. Moss de Oliveira, P.M.C. de Oliveira, and D. Stauffer, Evolution, Money, War and Computers (Teubner, Stuttgart-Leipzig, 1999)

[5] M.C. Gonzalez, A.O. Sousa, and H.J. Herrmann, Int. J. Mod. Phys. C 15, (2004) issue 1.

[6] J.C. Johnson, J.S. Boster and L.A. Palinhas, J. Mathematical Sociology 27 (2003) 89 